\title{
Data Encoding Improvement and Size Reduction of Chipless RFID Tag Using Short-Circuit Line Effect and Angle-Based Divided Loops
}

\author{
Nguyen Thanh Huong ${ }^{1^{*}}$, Tran Duc Quang ${ }^{2}$ \\ ${ }^{1}$ Hanoi University of Science and Technology, No. 1, Dai Co Viet str., Hai Ba Trung dist., Hanoi, Viet Nam \\ ${ }^{2} B K A V$ Electronics, BKAV Corporation, BKAV Building, Yen Hoa, Cau Giay, Hanoi, Vietnam \\ Received: August 05, 2020; Accepted: November 12, 2020
}

\begin{abstract}
This paper focuses on a short circuit and concentric loop effects to improve the structure dimension as well as enhance the ability to encode electromagnetic wave data for chipless RFID tags. The tag is composed of star-shaped rings that are concentrically nested together. The following factors help reduce the tag size: using the phenomenon of electromagnetic backscattering, assessment by the Radar Cross Section (RCS), the antenna-free tag only includes the multi-frequency resonators. By inserting short circuit lines combined with concentric loops, the number of resonant peaks increases exponentially without increasing the tag overall dimension. In addition, by inserting the short circuit lines at different angles between the two consecutive loops, we can adjust the resonant frequency in the frequency bands, thereby changing the value of the bit by shifting the frequency position. The overall dimension of the resulted tag is $13 \mathrm{~mm} \times 13 \mathrm{~mm}$ and the tag is aimed to be a printable tag on the flexible substrate to minimize the fabrication cost. With the 18-vertex star-shaped tag with five concentric loops, this chipless RFID tag can encode up to 16 bits with high resolution and large data density.
\end{abstract}

Keywords: Chipless RFID, angle division, short-circuit, frequency encoding, radar cross section

\section{Introduction}

Over the past few decades, several systems and technologies have been appeared and developed to identify and track the objects in producing systems and logistics. Among them, the most prominent are two technologies: Barcode (Barcode) and RFID (Radio Frequency Identification). Barcode utilizes a scanner with a beam of light to detect the black and white dots on a barcode. The scanner can decode the lines, turn them into text, and send the information to a host computer for later analysis or identify from its database. Barcode scanners have to "see" every line of the code to precisely read and transmit the data. Barcode technology has the advantage of small size and low price, but there are some drawbacks such as only one data code can be tested at a time, the code must be in direct contact (without obstacles) with a scanner or camera. RFID (Fig.1) is a technology that identifies objects by radio waves, hence it allows identification of objects through radio transceivers so that they can monitor and manage the objects that need to be surveyed. This is the advantage that makes RFID superior to barcode technology, which can identify objects at distances from a few meters to tens of meters in space.

\footnotetext{
* Corresponding author: Tel.: (+84) 904.466.684

Email: huong.nguyenthanh3@hust.edu.vn
}

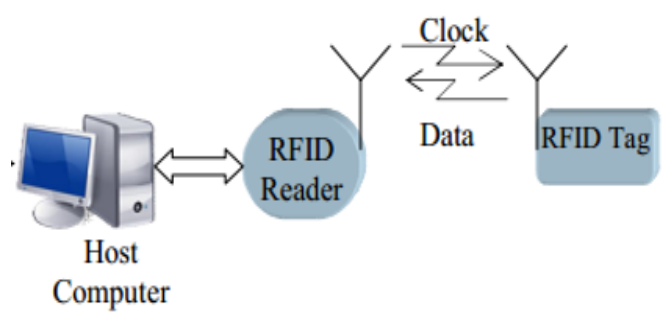

Fig. 1. Configuration of a conventional RFID system [1]

Besides the above advantages, the RFID system still has some disadvantages such as high cost due to the presence of the integrated chip on the tag [1]. Current RFID systems often comprise very large numbers of objects to identify and result in high costs for the entire system. To reduce costs for RFID systems, chipless RFID technology has been proposed, researched, and developed very strongly in recent years. This technology not only reduces costs by streamlining the usage of chips but also is made as simple as, for example, being able to print on inexpensive materials such as paper, plastic, and textile. In addition, the chipless RFID system employs passive tags therefore energy saving is also ensured. Chipless RFID technology is a low cost, effective, safe, and reliable solution [2].

Based on the data encoding method, the chipless RFID tag is divided into 3 types [3]: tags based on Time Domain Reflectometry (TDR-based) [4-8], tags based on natural spectral signature [9-10], tags based 
on amplitude-phase modulation [11-15]. Tags used are based on time-domain reflections, including printable and non-printable tags. A typical example of printable tags is a tag using Surface Acoustic Wave (SAW) [4-7]. SAW tags are excited by a Gaussian pulse transmitted by the reader. Due to the nature of the piezoelectric material, the tag cannot be printed on conventional paper or plastic materials. The tag proposed in this article is based on the time domain reflection and printable [8], using circuits to create transmission line latency.

The tags hinging on the natural feature of the operating data coding spectrum rely on the resonant properties of the structure. In the paper [9], chemical tags are designed from resonant filament deposition or special conductive ink. In the article [10], printed RFID tags apply electronic ink samples to be embedded or printed on the surface of the tagged objects to create the operating range up to $1.2 \mathrm{~m}$.

Chipless RFID tags based on amplitude and phase modulation are intensively studied tag. Examples of tags using this method are found in [11-14]. In the article [11], the tag consists of spiral resonators located near a microstrip line, each spiral resonating at a frequency and representing a bit, the size of the tag increases with the size of the bit. In order to encode a large number of bits, the tag size must be quite large. Tags encode data on both amplitude and phase using the "C" structure introduced in the article [12] and the ability to encode up to 22.9 bits on the tag size $2 \mathrm{~cm} \mathrm{x} 4 \mathrm{~cm}$. Using the dual polarization property of the wave, which doubles the ability to encode data, the square resonant structure is generated in the paper [13]. The structures are based on the letters of the alphabet in the article [14], in which each letter has its particular electromagnetic reflection properties, but the employment of these structures requires an investigation for each letter, as well as the ability to duplicate resonant frequencies.

The chipless RFID tag introduced in this paper uses electromagnetic wave backscattering, as measured by the Radar Cross Section (RCS) parameter. On the basis of the backscattering phenomenon, this tag does not require the antenna element to receive and transmit, so the size of the tag is significantly reduced. The tag structure also makes use of the concentric loop structure and short circuit line between the loops. In Section 2, the authors present the principle of electromagnetic wave backscattering, which is the main principle of structure. Section 3 describes the tag design procedure. In Section 4, the authors describe the simulation and measurement results. Lastly, the conclusion is drawn in Section 5 on tag performance.

\section{Theory of electromagnetic wave scattering}

For electromagnetic encryption technology, there are a number of principles and techniques used for encoding, but to satisfy the requirements of flat printable structure, small dimension, electromagnetic wave scattering principle is applied with the characteristics of specially featured elements at different frequencies.

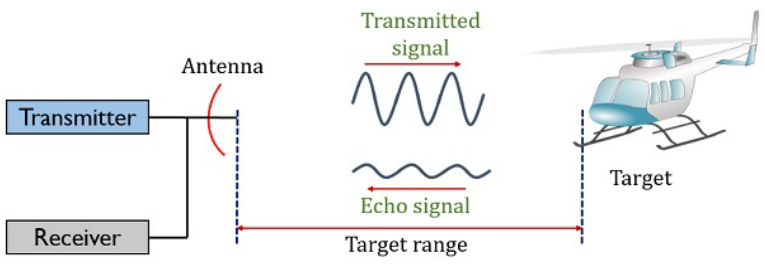

Fig. 2. Concept of Radar System [16]

Properties of this wave backscattering effect were studied according to the concept of a radar system (Fig.2) via the RCS (radar cross section $-\sigma$ ) of the desired object. Assuming that the power of incident wave to an object from radar at a distance of $\mathrm{R}$ is $P_{D i}$. Reflected power from the object is [15]:

$$
P_{r}=\sigma \cdot P_{D i}
$$

where $\sigma$ is the Radar Cross Section (RCS). Meanwhile, $P_{D r}$ is the backscattering power at the receiving antenna, we have:

$$
P_{D r}=\frac{P_{r}}{4 \pi R^{2}}
$$

From equations (1) and (2), the RCS is calculated as

$$
\sigma=4 \pi \mathrm{R} 2\left(\frac{P_{D r}}{P_{D i}}\right)
$$

To ensure that the receiving antenna is located at the far field (that means the backscattering wave received by the antenna is a plane wave), equation (3) is corrected to:

$$
\sigma=4 \pi \mathrm{R} 2 \lim _{R \rightarrow \infty}\left(\frac{P_{D r}}{P_{D i}}\right)
$$

The ability of the object to encode data is expressed through the number of RCS peaks (maximum backscattering power) in a given frequency range. If each RCS peak is considered a data bit, the criterion is that the encoding structure must create as many RCS peaks as possible to increase the encoding capacity, or the design structure must resonate at many different frequencies.

When projecting an excitation wave to an object, the received RCS versus frequency characteristic is divided into three regions: the lowfrequency region (Rayleigh region), the medium- 
frequency region (Resonance region), and the highfrequency region (Optical region) [17-18]. Fig.3. is an example of the RCS versus frequency characteristic of an ideal conductive sphere with a radius of $56 \mathrm{~cm}$. It is recommended that when designing a chipless RFID tag based on electromagnetic wave feedback, the tag should work in the Rayleigh region.

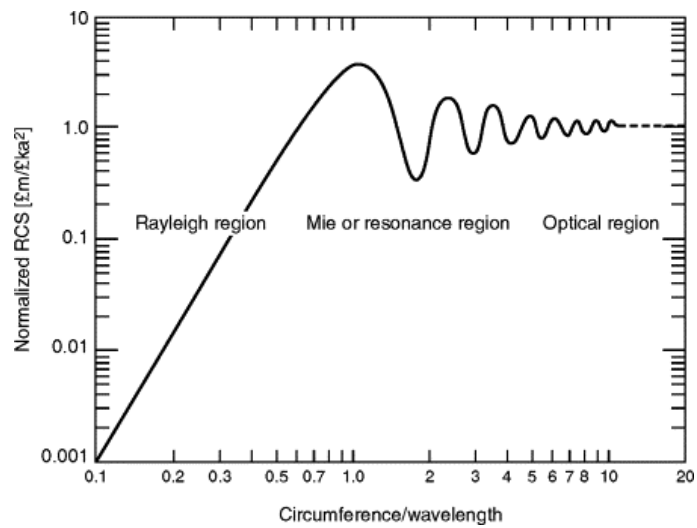

Fig. 3. RCS versus frequency of an ideal conducting sphere with a radius of $56 \mathrm{~cm}$ [17-18]

\section{Design of chipless RFID tag}

The tag is composed of 18-vertex star rings that are concentrically nested. Two consecutive stars form a loop, similar to the article [13]. The size of the loop is inversely proportional to the resonant frequency. The star structure makes it possible to increase the size of the loop on the same dielectric area as compared to the square [13], the U [2], the butterfly wings [3], from which the star structure will optimize data density over similar structures. By using short circuit lines to connect the vertices of the star rings, we divide a given star into smaller loops, hence each loop can generate a corresponding resonance.

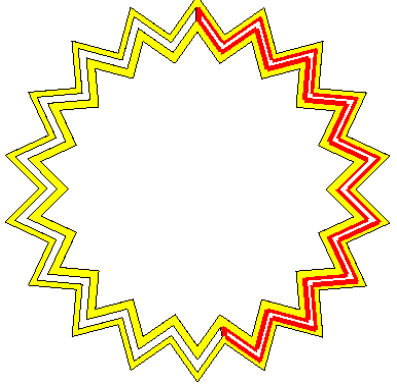

Fig. 4. The topology of the 18 -vertex star structure (the red line represents a loop)

First, we consider the bit encoding approach for each loop. Increasing the number of loops will extend the encryption capacity exponentially. Each large loop will be divided into two small loops of different lengths by using two short-circuit lines. These lines can be made on the top, the corner, and even the middle of the star edge. With the two short-circuit lines, the loop is divided into two smaller loops in which the length of two loops will be inferior to each other by an edge of the star (Fig.5a). These two small loops are adjusted to change the length by adding small short-cuts and do not change the length of the remaining loop (Fig.5b,c), thereby shifting the resonant frequency by one small interval. This length adjustment is done twice in order to adjust the one resonant frequency independently without affecting the remaining one by keeping the length of the loop unchanged.

Another technique to omit a resonant frequency without affecting the rest is to divide the individual nested loop into smaller ones (Fig.5d), thereby bringing this undesired frequency outside the frequency range of interest. In addition to the initial resonant frequency, the shifting of that frequency twice and finally disappear, we have the following encoding:

Table 1. Encoding resonant cases

\begin{tabular}{|l|c|}
\hline \multicolumn{1}{|c|}{ Cases } & Encoding \\
\hline No resonant peak detected & 00 \\
\hline Resonant peak detected & 01 \\
\hline Resonant peak first shift & 10 \\
\hline Resonant peak second shift & 11 \\
\hline
\end{tabular}

In a nutshell, from a large loop originally made from two stars in combination with the abovementioned shifting techniques, we are able to encode a total of 4 bits. Furthermore, tuning the resonant frequency was made easily by inserting short-circuit lines. In general, for $N$ star circles, the number of encoding bits obtained will be $4(\mathrm{~N}-1)$ bits.

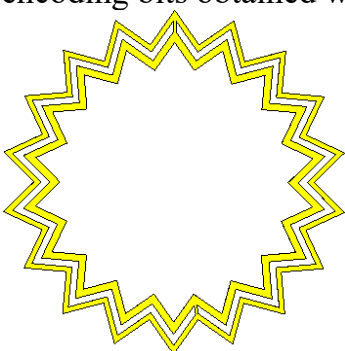

a)

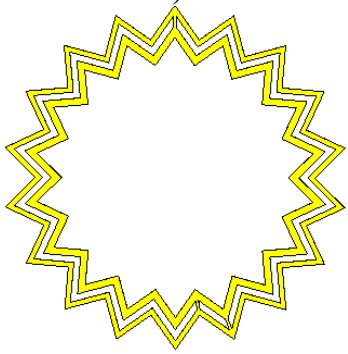

c)

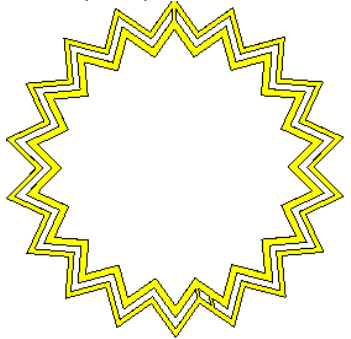

b)

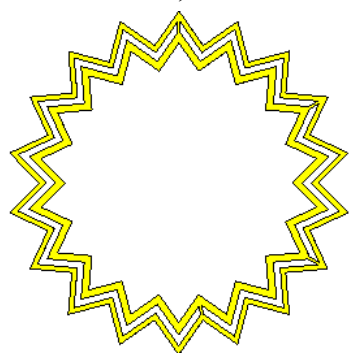

d)
Fig. 5. Short-circuit line cases used in the encoding process of 18-vertex star structures 


\section{Results and discussion}

The structure is designed on Polyimide material with the dimension of $13 \mathrm{~mm} \times 13 \mathrm{~mm} \times 0.1 \mathrm{~mm}$ with a dielectric constant of 3.5 , tan $\delta$ loss coefficient of 0.0027 . The simulation results for the structures of Fig.5 are shown in Fig.6.

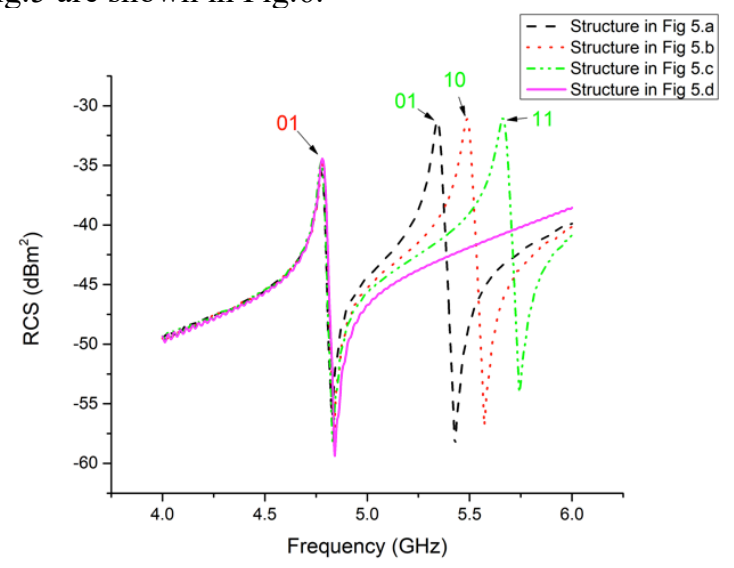

Fig. 6. Simulation results of Fig. 5 structure

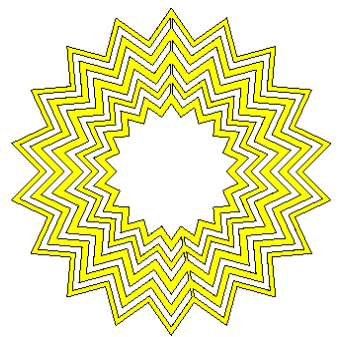

a)

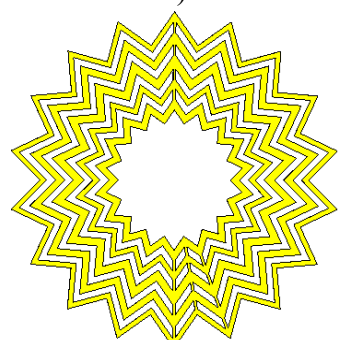

c)

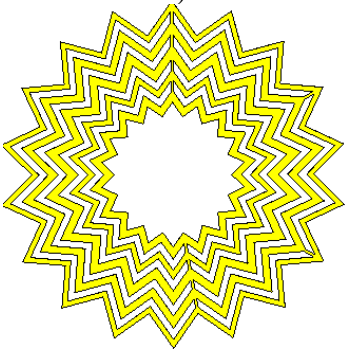

e)

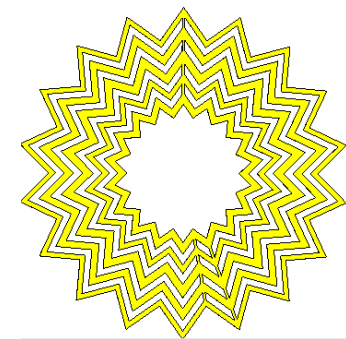

b)

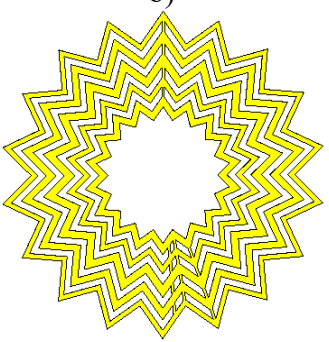

d)
Fig. 7. Star-shaped chipless tag encoding structure with 18 vertices and 5 concentric loops

Next, we will use five-star loops to form 4 electrical loops, thereby increasing the number of bits to $4 \times 4=16$ bits. The number elevation of star rings does not enlarge the size of the tag. On the contrary, the encoding ability of the loops is done completely independently without affecting the encryption of the remaining loops. The length of the loops is adjusted to avoid overlapping resonance peaks by changing the size of the star rings. The tag design structures according to this approach are shown in Fig.7.

On selecting the design of Fig.7a as a reference, each large loop is divided into two smaller loops, equivalent to the 16-bit encoding is 0101010101010101. In Fig.7b, all the small loops are reduced in length by two times, which causes all of the greater resonant frequencies of the loops to shift by two-bit positions, equivalent to coding 0111011101110111. Meanwhile, in Fig.7c all large and small loops are translated to be reduced by two times, equivalent to with encoding 1111111111111111. In Fig.7d, all loops of greater length are reduced by length once and all smaller loops are reduced by length twice, the equivalent encoding is 1011101110111011 . By breaking up the large outer loop, we erase the maximum resonant frequency, thereby creating the code 0001010101010101 as illustrated in Fig.7e. Simulation results for RCS value in all cases of Fig.7 are exhibited in Fig.8. By encoding 16 bits on a $13 \times 13 \mathrm{~mm}^{2}$ tag area, we obtain a data density of $9.47 \mathrm{bits} / \mathrm{cm}^{2}$, which is larger than the previously researched chipless RFID tag structures [4-12] $[19,20]$.

A table that shows the comparison among the encoding capability of the proposed structure with some of the existing structures is displayed in Table 2. Other tag structure parameters are taken from the research in papers [12], [19,20].

Table 2. Comparison of our proposed tag with previously studied chipless RFID tag

\begin{tabular}{|l|c|c|c|}
\hline Tag structure & $\begin{array}{c}\text { Data } \\
\text { Density } \\
\text { (bit/cm }\end{array}$ & Printability & $\begin{array}{c}\text { Tag dimension } \\
\text { increased with the } \\
\text { number of bits }\end{array}$ \\
\hline SAW & $>1$ & $\mathrm{~N}$ & $\mathrm{Y}$ \\
\hline Delay line & $>0.17$ & $\mathrm{Y}$ & $\mathrm{Y}$ \\
\hline Chemical & 1.76 & $\mathrm{Y}$ & $\mathrm{N}$ \\
\hline Ink tattoo & $>1$ & $\mathrm{Y}$ & $\mathrm{N}$ \\
\hline $\begin{array}{l}\text { Spiral } \\
\text { Resonator }\end{array}$ & 0.61 & $\mathrm{Y}$ & $\mathrm{Y}$ \\
\hline $\begin{array}{l}\text { C- like hybrid } \\
\text { coding tag [12] }\end{array}$ & 2.86 & $\mathrm{Y}$ & $\mathrm{Y}$ \\
\hline $\begin{array}{l}\text { Butterfly Slot } \\
\text { tag [20] }\end{array}$ & 5.1 & $\mathrm{Y}$ & $\mathrm{N}$ \\
\hline $\begin{array}{l}\text { Dual Polarized } \\
\text { Slot U tag [19] }\end{array}$ & $>7$ & $\mathrm{Y}$ & $\mathrm{N}$ \\
\hline Our tag & $\mathbf{9 . 4 7}$ & $\mathrm{Y}$ & $\mathbf{N}$ \\
\hline
\end{tabular}


The results in Fig.8a-e correspond to the structure in Fig.7a-e, respectively. In Fig.8a, the RCS peaks obtained at 8 frequencies and the distance between two consecutive peaks are quite evenly spaced, hence this tag is regarded as the reference tag with the bit data of 0101010101010101. When inserting the short-circuits to the star loops in the reference tag as in Fig. $7 \mathrm{~b}$, it can be seen in Fig. $7 \mathrm{~b}$ that the frequency from $5.36 \mathrm{GHz}$ is shifted to $5.69 \mathrm{GHz}$, the peak at $6.62 \mathrm{GHz}$ is shifted to $7.01 \mathrm{GHz}$, the peak at $8.18 \mathrm{GHz}$ is shifted to $8.65 \mathrm{GHz}$ and the peak at $10.25 \mathrm{GHz}$ is shifted to $10.84 \mathrm{GHz}$ while the other four peaks remained the same, just shifted a little bit due to the mutual coupling change when adding the small short-circuit lines. This shifting results in a new tag with the new encoded data of 0111011101110111 . In Fig.7c, short circuits are continuously inserting to Fig.7b and the similar shifting happens, which leads to the new tag data of 1111111111111111 . This rule is applied even when we change the position of the first set of shortcircuits compared with the reference tag. The arrangement of Fig.7d is modified from Fig.7c where he entire set of reference peaks as in Fig.7a shift to

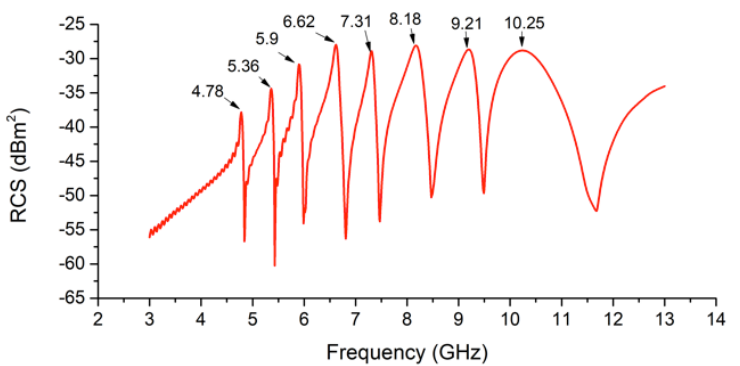

a)

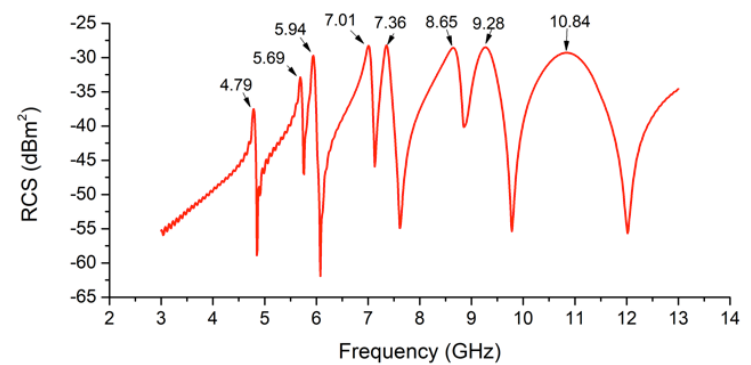

b)

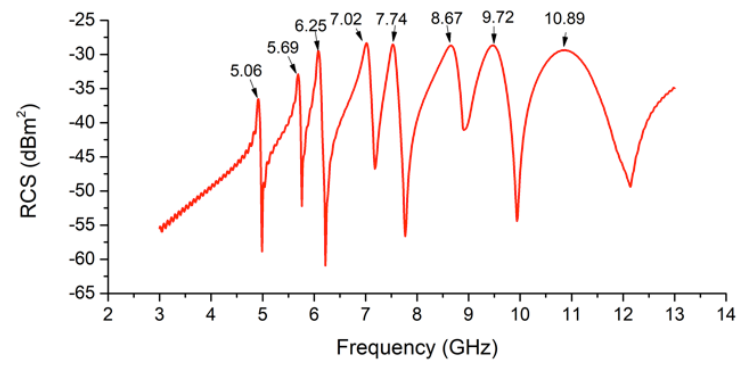

c) make the data switch to 1011101110111011. Lastly, in Fig.7e, we erase the maximum resonant frequency, and the new data of 0001010101010101 is generated resultantly.

Consequently, based on the ability to change the position of the short circuits on a circle associated with the concentric ring structure, our proposed chipless RFID tag has gained a remarkable encryption data density compared with the majority of tag structures that have been studied before. Owing to the backscattering principle, the tag does not need an antenna on its structure while can still receive the signal from the reader. In responding to the interrogation signal from the reader, the tag can reflect the encoded signal. The receiver will receive frequency-coded signals from the card. With a good resolution (the discrimination between the two consecutive RCS peaks) and the clear difference between the maximum and minimum RCS response, the feedback signals from different tags are independent of each other and can be totally adjusted without affecting each other's frequency.

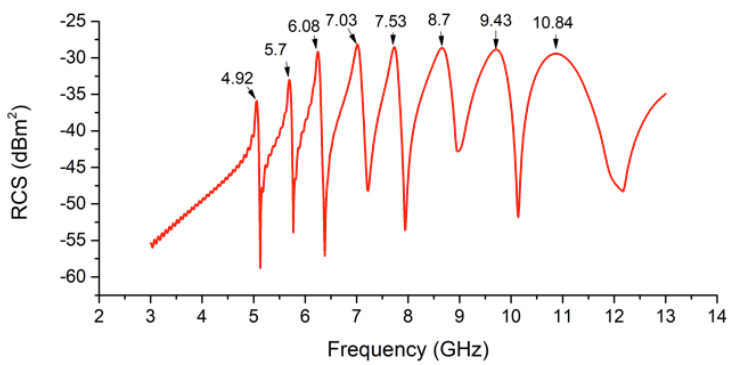

d)

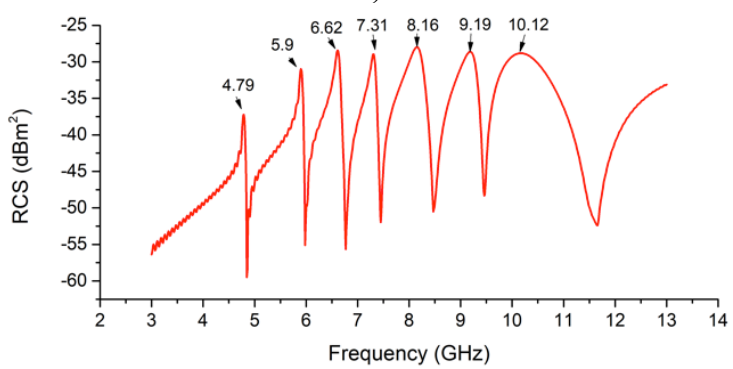

e)

Fig. 8. Simulation results of RCS versus frequency of star-shaped chipless tag encoding structure with 18 vertices and 5 concentric loops in Fig. 7. 


\section{Conclusion}

In this article, a 16-bit star-shaped chipless RFID tag which is designed based on wave backscattering is evaluated using RCS. The tag is fully printable and has a compact size. The tag structure consists of concentrically nested stars, so when you want to increase the number of bits, you only need to increase the number of stars, not increasing the size of the tag. The tag used is a magnetic card thus resistant to the effects of the environment. By adding short circuits between the two nearby stars, we adjust the appearance of the bits, which helps to change the ID of each tag in a flexible way.

\section{Acknowledgments}

This research is funded by the Hanoi University of Science and Technology (HUST) under project number T2020-PC-019.

\section{References}

[1] Preradovic, S. and Karmakar, N.C., 2010. Chipless RFID: Bar code of the future. IEEE Microwave Magazine, 11(7), pp.87-97.

[2] Das, R., 2006. Chip-less RFID-The end game. IDTechEx. Cambridge, MA.

[3] C. Feng, C., Zhang, W., Li, L., Han, L., Chen, X. and Ma, R., 2015. Angle-based chipless RFID tag with high capacity and insensitivity to polarization. IEEE Transactions on Antennas and Propagation, vol. 63, no. 4, pp.1789-1797.

[4] Harma, S., Plessky, V.P., Li, X. and Hartogh, P., 2009. Feasibility of ultra-wideband SAW RFID tags meeting FCC rules. IEEE Transactions on ultrasonics, ferroelectrics, and frequency control, 56(4), pp.812820.

[5] Han, T., Wang, W., Wu, H. and Shui, Y., 2008. Reflection and scattering characteristics of reflectors in SAW tags. IEEE Transactions on ultrasonics, ferroelectrics, and frequency control, 55(6), pp.13871390.

[6] Saldanha, N. and Malocha, D.C., 2007, October. P4J-1 design parameters for SAW multi-tone frequency coded reflectors. In 2007 IEEE Ultrasonics Symposium Proceedings (pp. 2087-2090).

[7] Liu, J. and Yao, J., 2008. Wireless RF identification system based on SAW. IEEE Transactions on industrial electronics, 55(2), pp.958-961.
[8] Vemagiri, J., Chamarti, A., Agarwal, M. and Varahramyan, K., 2007. Transmission line delay-based radio frequency identification (RFID) tag. Microwave and optical technology letters, 49(8), pp.1900-1904.

[9] Glickstein, M., Feb. 2004. Firewall protection for paper documents. RFID Journal internet article.

[10] Jones, K.C., 2007. Invisible tattoo ink for chipless RFID safe, company says. EE Times white paper.

[11] Amin, E.M. and Karmakar, N., 2011, October. Development of a chipless RFID temperature sensor using cascaded spiral resonators. In IEEE Sensors, 2011 (pp. 554-557).

[12] Vena, A., Perret, E. and Tedjini, S., 2011. Chipless RFID tag using hybrid coding technique. IEEE Transactions on Microwave Theory and Techniques, 59(12), pp.3356-3364.

[13] Huang, H.F. and Su, L., 2016. A compact dualpolarized chipless RFID tag by using nested concentric square loops. IEEE Antennas and Wireless Propagation Letters, 16, pp.1036-1039.

[14] Tedjini, S., Boularess, O., Andriamiharivolamena, T., Rmili, H. and Aguili, T., 2017, June. A novel design of chipless RFID tags based on alphabets. In 2017 IEEE MTT-S International Microwave Symposium (IMS) (pp. 1561-1563).

[15] Mahafza, B.R., 2002. Radar systems analysis and design using MATLAB. CRC press.

[16] https://electronicsdesk.com/radar-system.html

[17] Uluisik, C., Cakir, G., Cakir, M. and Sevgi, L., 2008. Radar cross section (RCS) modeling and simulation, part 1: a tutorial review of definitions, strategies, and canonical examples. IEEE Antennas and Propagation Magazine, 50(1), pp.115-126.

[18] Li, H.J. and Kiang, Y.W., 2005. Radar and inverse scattering. In The Electrical Engineering Handbook (pp. 680-682). Elsevier.

[19] Islam, M.A. and Karmakar, N.C., 2012. A novel compact printable dual-polarized chipless RFID system. IEEE Transactions on Microwave Theory and Techniques, 60(7), pp.2142-2151.

[20] Riaz, M.A., Abdullah, Y., Shahid, H., Amin, Y., Akram, A. and Tenhunen, H., 2018. Novel butterfly slot based chipless RFID tag. Radioengineering, 27(3), p.777. 